\title{
DOSSIER
}

\section{Breve panorama histórico de la educación intercultural en América Latina}

\section{A Brief Historical Overview of Intercultural Education in Latin America}

\author{
Jocelin Venegas Martínez \\ Universidad Iberoamericana Ciudad De México, México \\ jocelin.venegas@ibero.mx \\ Luz María Stella Moreno Medrano \\ Universidad Iberoamericana Ciudad De México, México \\ luzmaria.moreno@ibero.mx
}

8

\section{RESUMEN}

Este artículo presenta un breve panorama histórico de la educación intercultural en América Latina desde los primeros ańos del siglo xx hasta el momento actual, con el objetivo de analizar la evolución del concepto en relación con las principales políticas públicas que se han implementado en diversos países de la región. El objetivo es brindar al lector una revisión general de las luchas sociales que han acompañado la educación intercultural en América Latina con el fin de conocer el contexto social e histórico que lo distingue de otros modelos educativos, como la educación multicultural, la educación ciudadana y la inclusión, entre muchos otros. La educación intercultural crítica y decolonial es, en el siglo XXI, una apuesta por recuperar una educación desde abajo y con una perspectiva histórica que toma como punto de partida los movimientos de resistencia de los pueblos indígenas del continente.

Palabras clave: Educación indígena; educación interculturalidad crítica; educación interculturalidad funcional; educación decolonial.

\begin{abstract}
This article presents a brief historical review of the intercultural education in Latin America in the 20th century until today, with the objective of analyzing the evolution of the concept along with the main public policies in every decade in several countries of the region. It aims to present the reader a general review of the social struggles associated to intercultural education in Latin America with the objective of understanding the socio-historical context that distinguishes between intercultural and multicultural education, inclusion and citizen education, among others. Critical and decolonial education is in the 21st century a chance to mend an education from the bottom with a historical perspective that has as a starting point the movements of resistance of the indigenous peoples of the continent.
\end{abstract}




\section{Keywords: Indigenous Education; Critical Intercultural Education; Functional Intercultural Education; Decolonial Education.}

Fecha de recepción: 20/10/2019

Fecha de aceptación: 18/01/2020

\section{Introducción}

La gran diversidad cultural, étnica y lingüística de América Latina constituye, sin duda, la mayor riqueza de esta región del continente. En América Latina, o Abya Yala, habitan 522 pueblos indígenas y se hablan 420 lenguas distintas (Unicef, 2009). Esto representa aproximadamente 42 millones de personas, es decir, $8 \%$ de la población total de la región. ${ }^{1}$ Sin embargo, desde la ocupación y la colonización europea de América, la historia de reconocimiento de los pueblos indígenas está plagada de contradicciones y negación de derechos bajo diversas formas, unas más veladas que otras. Desde la colonización, se llevó a cabo una clasificación jerárquica y racializada de las poblaciones originarias, y en el momento de la consolidación de los Estados nación latinoamericanos era necesario unificar lenguas, ideologías, valores y creencias. En este sentido, los pueblos indígenas fueron obligados a asimilarse $y$, en muchos casos, pueblos enteros fueron incluso exterminados por ser considerados inferiores a la cultura hegemónica dominante (López, 2001).

Para lograr este objetivo, el sistema educativo ha jugado un papel protagónico como herramienta para la homogeneización lingüística y cultural. La escuela se convirtió en una institución que contribuye "no sólo a formar en lo intelectual sino también de forjar las identidades ciudadanas" (Briceño, 2019, p. 117). Fue hasta finales del siglo xx cuando empezó a considerarse la diversidad cultural como un motivo de orgullo nacional. Esto implicó que varios países con población indígena reconocieran en sus constituciones la pluriculturalidad que los caracteriza y así la educación intercultural comenzó a tomar fuerza en todo el mundo (Schmelkes, 2004).

De este modo, muchos países empezaron a "plantear un proyecto educativo sensible a las diferencias culturales y a la inquietud que tales diferencias introducen en el proceso educativo: esto es, un proyecto de educación intercultural" (Díaz-Couder, 1998, p. 12). En muchos sentidos, este logro se dio gracias a la lucha sostenida de los movimientos indígenas por el reconocimiento de sus derechos entre los cuales se encuentra el de una educación que considere su cultura y sea pertinente a su contexto-, lo que despertó una mayor conciencia internacional de la "deuda histórica resultante de la colonización y de la consecuente subalternidad" de las poblaciones indígenas (López, 2001, p. 2). ${ }^{2}$ Tales ejemplos de lucha pueden verse en la actualidad como movimientos de resistencia étnica en diversos países del continente, como el Consejo Regional Indígena del Cauca (CRIC) en Colombia, la Coordinadora de las Organizaciones Indígenas de la Cuenca Amazónica (COICA) en Ecuador, la Asociación Interétnica de Desarrollo de la Selva Peruana (AIDESEP) en Perú y el Ejército Zapatista de Liberación Nacional (EZLN).

A partir del reconocimiento del conflicto histórico entre la cultura dominante y las culturas minoritarias, ${ }^{3}$ y con el fin de caminar hacia la construcción de un mundo más democrático en el que sea posible convivir entre diferentes, en muchos países latinoamericanos se apuesta por una educación intercultu-

\footnotetext{
${ }^{1}$ Estas cifras son resultado de censos realizados en América Latina por diferentes organismos, entre los que se encuentra la Comisión Económica para América Latina y el Caribe (CEPAL), la Organización de las Naciones Unidas para la Educación, la Ciencia y la Cultura (Unesco), la Organización Mundial de la Salud (oms), entre otros. Cabe mencionar que éstos no tienen un criterio uniforme respecto a lo que se considera como indígena.

${ }^{2}$ Bajo esta atmósfera, aparecen varios marcos jurídicos que tienen como propósito defender y velar por los derechos de los pueblos indígenas y preservar la cultura, por ejemplo, el Convenio 169 de la Organización Internacional del Trabajo (огт) sobre pueblos indígenas o la Declaración de los Derechos Humanos para los Pueblos Indígenas.

${ }^{3}$ Aunque las luchas de las comunidades negras y afrodescendientes no son muy mencionadas en la literatura, lo cierto es que desde hace mucho tiempo, al igual que los pueblos indígenas, han llevado a cabo movimientos por condiciones de vida más dignas y por el combate a la discriminación y el racismo, buscando con ello el reconocimientos de sus identidades culturales (López, 2001, p. 3; Ferrão, 2010, p. 346).
}

78 - Breve panorama histórico de la educación intercultural en América Latina Jocelin Venegas Martínez, Luz Marías Stella Moreno Medrano. DIDAC 76 (2020): 77-85 
ral que implique una relación comprensiva y respetuosa entre personas y grupos desde una posición de igualdad, en la que las culturas pueden enriquecerse entre sí. Esto contrasta con la educación multicultural, promovida sobre todo en países anglosajones, que está a favor de la coexistencia de personas o grupos de culturas diferentes en espacios o territorios determinados, pero que no necesariamente plantea un cambio en las relaciones de poder ni el reconocimiento de los derechos de las minorías (Schmelkes, 2003, 2004).

Sin embargo, a pesar de que la educación intercultural intenta brindar una perspectiva diferente, una vez que se ha institucionalizado sigue una implementación muy similar al multiculturalismo en América Latina. Los países que han logrado cuestionar de manera más profunda estos modelos son Bolivia y Ecuador, y han podido implementar políticas de reconocimiento a los pueblos indígenas.

Bajo tal contexto, este artículo pretende mostrar, a partir del siglo xx, la trayectoria histórica de Abya Yala en la búsqueda de modelos educativos que reconozcan y respeten su diversidad, con el fin de conocer la diferencia sustantiva entre las perspectivas más fuertes de la interculturalidad — la funcional y la crítica-y cómo ambas tienen consecuencias dis- tintas en los planes y las políticas educativas. Finalmente, se abordará la interculturalidad crítica como un proceso necesario para la construcción de un mundo más justo y equitativo, en el que la pedagogía decolonial adquiere un papel fundamental.

\section{Breve recorrido histórico de la educación indigena}

Las primeras experiencias de educación bilingüe en América Latina fueron promovidas en el siglo XVI por los misioneros, quienes a través de folletos $\mathrm{y}$ abecedarios mimeografiados introdujeron a los pueblos indígenas al uso de la lengua escrita (Abram, 2004). Otra iniciativa interesante a principios del siglo xx fue encabezada entre 1910 y 1930, por José Antonio Encinas, quien realizó observaciones sobre los procesos de aprendizaje de quechuas y aymaras que asistían a su Centro Educativo en Puno, Perú, y planteó la necesidad de partir de la cultura y lengua indígena para crear cualquier proyecto educativo, además de buscar que la educación respondiera a las problemáticas de sus regiones (López, 1998).

Sin embargo, experiencias como éstas fueron sumamente aisladas y, por lo tanto, tuvieron poca incidencia. La tendencia predominante en diversos países latinoamericanos era la erradicación total de lo indígena en la formación escolar, ejemplo de esto
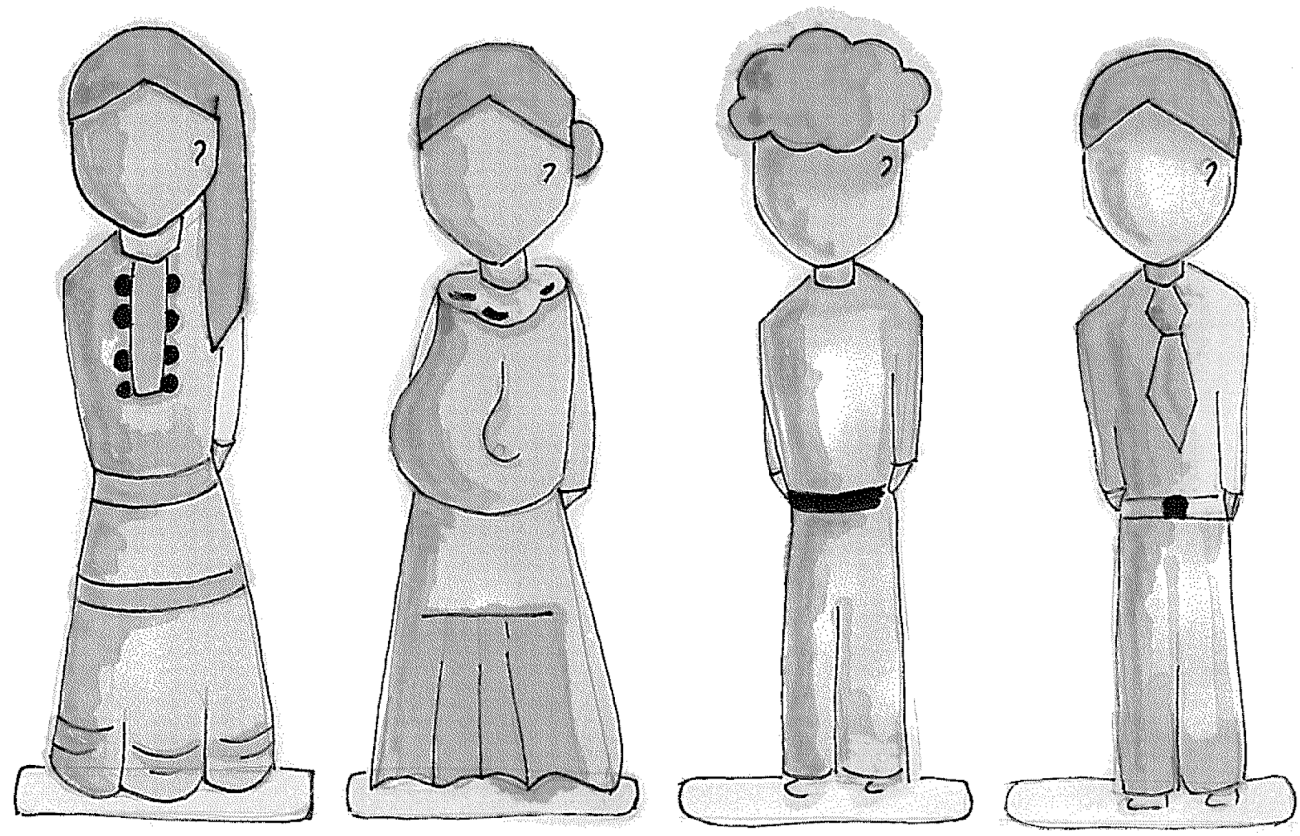

Breve panorama histórico de la educación intercultural en América Latina • Jocelin Venegas Martínez, Luz Marías Stella Moreno Medrano. DIDAC 76 (2020): 77-85 


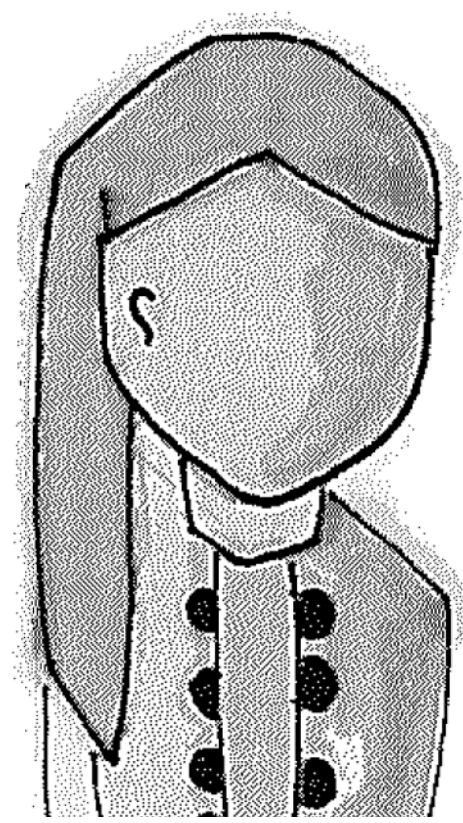

es la ley de educación rudimentaria promulgada en México en 1911, en la cual se prohibía el uso de las lenguas maternas y se proponían diversos métodos de castellanización (López, 1998).

Esta etapa se caracterizó por ser sumamente violenta y etnocentrista, pues pretendía imponer la cultura hegemónica dominante sobre los pueblos originarios (Ajagán, 2017; Ferrão, 2010) a través de la homogeneización lingüístico-cultural. El mecanismo utilizado para lograr esta meta fue, entre otras cosas, la implementación de campañas de castellanización (López, 2001). Asimismo, surgieron las primeras escuelas bilingües en las que, a la par de la castellanización, se hizo uso de una estrategia asimilacionista, en la que se utilizaban las lenguas nativas para "alfabetizar y civilizar más fácilmente a pueblos enteros” (Ferrão, 2010, p. 345). Esta concepción del bilingüismo influyó en las políticas educativas dirigidas a los indígenas y se mantuvo hasta la década de 1960 .

En México, una experiencia importante en ese sentido fue la Casa del Estudiante Indígena, funda- da en 1925, con el propósito de formar a jóvenes indígenas como intermediarios culturales entre la nación y sus comunidades con la lengua materna como medio de transmisión educativa (Loyo, 1996; López, 1998). También en Perú se realizaron estudios en torno a la escritura en quechua y aymara, y su utilización en la educación de la población hablante de esas lenguas. Sin embargo, los hallazgos de estas y otras iniciativas son poco conocidos y difundidos (Chirinos \& Zegarra, 2004).

En la tercera década del siglo xx, un evento importante para la educación bilingüe en Latinoamérica fue la llegada del Instituto Lingüístico de Verano (ILv). ${ }^{4}$ En el caso particular de México, Lázaro Cárdenas apoyó su implementación con la finalidad de alfabetizar y castellanizar a los pueblos indígenas que eran vistos como un factor de retraso para la modernización del país. De esta forma, se fortaleció aún más el modelo asimilacionista, implementado a través de una educación bilingüe de transición en la que las lenguas indígenas son consideradas un "mal necesario" para lograr la unidad nacional (López, 1998). Sin embargo, contrario al objetivo del ILV, algunos grupos indígenas consideraron que el dominio de la lengua predominante, el español, era una herramienta para luchar por sus derechos y organizarse, y además buscaron desarrollar una conciencia étnica crítica para legitimar su cultura (Fajardo, 2011). Al mismo tiempo, se comenzaron a desarrollar proyectos autogestivos encabezados por docentes en los que se implementaron metodologías bilingües innovadoras para evitar el uso exclusivo del español, que era una limitante para el aprendizaje de aquellos estudiantes indígenas monolingües. Iniciativas como éstas tuvieron lugar en Ecuador, México, Bolivia y Perú (Fajardo, 2011).

En 1938 tuvo lugar la Octava Conferencia Internacional Panamericana realizada en Lima, en la que se recomendó la creación del Instituto Indigenista Interamericano (III), hecho que se concretó en

\footnotetext{
${ }^{4} \mathrm{El} \mathrm{ILv}$ era una organización estadounidense de corte religioso y conservador que "mediante el mensaje salvacionista, trata de imponer un modelo de dominación cultural que acreciente el individualismo, el apoliticismo, el conformismo y una actitud pasiva ante los problemas sociales” (Santoyo \& Arellano, 1997, p. 234). Esta organización también tuvo presencia en Colombia, Perú, Ecuador y otros países latinoamericanos. Para 1936 trabajaban con los nahuas, otomíes, huicholes, tarahumaras, mayas, tarascos, mazatecos, mixes, mixtecos, totonacas, entre otros.racismo, buscando con ello el reconocimientos de sus identidades culturales (López, 2001, p. 3; Ferrão, 2010, p. 346).
}

80 - Breve panorama histórico de la educación intercultural en América Latina Jocelin Venegas Martínez, Luz Marías Stella Moreno Medrano. DIDAC 76 (2020): 77-85 
Pátzcuaro en 1940. Los objetivos principales del III fueron la colaboración de los Estados miembros en la creación de políticas indigenistas, así como la promoción de los trabajos de investigación y la capacitación de aquellas personas que se dedicaban al trabajo comunitario en zonas indígenas. La labor más importante del III fue destacar la importancia de la alfabetización en la lengua materna y la creación de proyectos de educación bilingüe. Asimismo, el III fue un medio para señalar y denunciar las problemáticas de los pueblos indígenas en el continente, como la discriminación, el racismo y la falta de acceso a servicios médicos, entre muchos otros derechos (Zolla \& Zolla Márquez, 2010).

En la década de 1970, comenzaron a surgir iniciativas que buscaban trascender tales experimentos institucionales asimilacionistas para integrar a los diversos grupos indígenas a la sociedad nacional. Dichas iniciativas fueron promovidas por antropólogos, indigenistas, teólogos y catequistas provenientes de la teología de la liberación, solidarios con las luchas por los derechos sociales. Destaca, entre ellas, el trabajo de Paulo Freire en Brasil, el cual abrió una ventana más para incorporar los contextos socioculturales en los procesos educativos. A través de la crítica a la educación bancaria y su método de alfabetización para entender la realidad social y promover la organización comunitaria, muchos proyectos educativos reconocieron y explicitaron su dimensión política. A diferencia de las políticas educativas institucionales que, hasta ese momento, se habían gestado desde la oficialidad, Freire, entre otros muchos pedagogos latinoamericanos, puso en el centro del debate la lucha de clases y la importancia de analizar críticamente los procesos de colonización como parte de una pedagogía liberadora. Estas ideas tienen una fuerte influencia en la conformación de la llamada "pedagogía decolonial" que, como veremos más adelante, apuesta por prácticas educativas emancipadoras, cuyo propósito es la transformación crítica de la realidad social.
En nuestro continente, durante la década de 1970, comienza a discutirse la necesidad de garantizar derechos colectivos diferenciados de los pueblos indígenas, entre ellos la educación pertinente y respetuosa a la diversidad sociocultural y lingüística (Sartorello, 2009). Las Declaraciones de Barbados I y II abonaron a esta idea, pues gracias a ellas "surgió un fructífero debate ideológico y epistemológico sobre los derechos a la autodeterminación de los pueblos indoamericanos concernientes a su vida comunitaria como gobernanza, salud, educación y protección de los recursos naturales de sus territorios” (Briceño, 2019, p. 115). Con base en esta visión, se rompió con la concepción del bilingüismo como herramienta dirigida exclusivamente a la integración de los indígenas a la cultura hegemónica, pues plantea que no sólo el uso de la lengua propia es fundamental para que los pueblos indígenas mantengan y fortalezcan su cultura, sino que también es de vital importancia reconocer los saberes ancestrales y los valores tradicionales de las culturas indígenas en el currículum escolar (Fajardo, 2011).

Un producto de estos avances de la educación indígena fue la búsqueda de los saberes ancestrales y, bajo este contexto, surgió la educación bilingüe bicultural (ЕВв) que, a pesar de reconocer dos culturas y dos lenguas, sostiene la perspectiva de que una siempre será la dominante (Fajardo, 2011, p. 19). En los hechos, la EBB terminó caracterizándose por tener una visión folclorista de los pueblos indígenas en la que, tal y como sostiene Moya (1998), el biculturalismo continuó limitándose a un modelo de transición y asimilación cultural.

En las décadas de 1980 y 1990, comenzaron a crearse marcos legales que otorgaron a los pueblos originarios protección e igualdad ante la ley, gracias a las múltiples luchas encabezadas por pueblos indígenas, ${ }^{5}$ afrodescendientes, así como movilizaciones magisteriales y reivindicaciones comunitarias (López, 2001; Walsh, 2005; Schmelkes, 2004). De esta manera, el reconocimiento jurídico empezó a

\footnotetext{
${ }^{5}$ Un ejemplo emblemático de la fuerza de un movimiento indígena es el levantamiento del ezLn el 1 de enero de 1994 en Chiapas. A través de éste, se logró visibilizar a nivel mundial la presencia de población indígena en México, pero también las condiciones de pobreza, inequidad y la merma en derechos civiles en la que han vivido (Briceño, 2019, p. 116).
} 
constituirse como un logro político de la sociedad civil ${ }^{6}$ (Apodaca, 2009; Dietz, 2012). Sin embargo, otros autores señalan que este fenómeno también es resultado de la inminente entrada del proyecto neoliberal a América Latina, mismo que se presenta bajo el eslogan de considerar a los sectores históricamente excluidos a manera de estrategia para mantenerlos "apaciguados". A esta nueva fase político-social se le conoce como constitucionalismo multicultural o multiculturalismo constitucionalista (López, 2001; Walsh, 2010; Ferrão, 2010; Ajagán, 2017).

Dentro de esta coyuntura aparece por primera vez en América Latina el término "interculturalidad". ${ }^{7}$ Esto ocurre en una reunión de especialistas realizada en México en la que se discutió la importancia de establecer políticas nacionales de plurilingüismo y multietnicidad y proponiendo, entre otras cosas, la oficialización nacional y regional de lenguas indígenas y políticas educativas globales (Walsh, 2010). Así, comienzan a observarse cambios encaminados hacia la interculturalidad en la educación en diversos países como Colombia, Ecuador, Perú, Bolivia, Nicaragua, Guatemala, entre otros (Bertely, 2013).

Sin embargo, como todo proceso de cambio profundo, no ha estado exento de contradicciones, avances y retrocesos. Ya en los inicios del siglo XXI, la educación dirigida a los pueblos indígenas, ahora denominada intercultural bilingüe (ЕIB), sigue caracterizándose por centrar el uso de la lengua en una sola direccionalidad: de la lengua indígena a la lengua nacional. De esta manera, la lengua sigue siendo una herramienta de transición hacia la adaptación y asimilación de los pueblos indígenas (Walsh, 2010). La еІв ha sido insuficiente para promover un justo intercambio cultural y, sobre todo, un equilibrio en las relaciones de poder entre indígenas y no indígenas. Los derechos de los pueblos indígenas siguen siendo transgredidos y hay una sistemática y permanente imposición de valores de la cultura dominante sobre los distintos pueblos (Ferrão, 2010).

\section{Interculturalidad funcional o interculturalidad critica}

Hay una gran disonancia entre el discurso y la práctica en las políticas públicas latinoamericanas. En la mayoría de los países se reconoce la necesidad de

romper con la historia hegemónica de una cultura dominante y otras subordinadas, y de esa manera, reforzar las identidades tradicionalmente excluidas para construir, en la vida cotidiana, una convivencia de respeto y de legitimidad entre todos los grupos de la sociedad (Walsh, 1998, p. 3).

Sin embargo, como Bertely menciona, "en cada país las funciones y los sentidos epistémicos y didácticos atribuidos al mismo no son unívocos" (2013, p. 43), es decir, existen diferentes miradas desde las que se considera que puede y debe construirse la interculturalidad. De esta manera, existen dos formas desde las que se construyen los discursos políticos y pedagógicos de la educación intercultural en América Latina: la interculturalidad funcional y la interculturalidad crítica. La principal diferencia entre ambas posturas "es la visibilización o el desdibujamiento del conflicto" que ocurre entre diversos grupos culturales que se encuentran en desiguales condiciones de poder (Apodaca, 2009, p. 6).

Desde una perspectiva funcional, la interculturalidad sirve al sistema dominante, pues "el reconocimiento y el respeto a la diversidad cultural se convierte en una nueva estrategia de dominación" (Walsh, 2010, p. 3) que busca mantener la estabilidad social a través del control del conflicto étnico, con la finalidad de perpetuar el sistema neoliberal y con esto las desigualdades sociales. Así, la educación intercultural funcional no promueve la construc-

\footnotetext{
${ }^{6}$ El Convenio 169 de la oIT sobre pueblos indígenas y tribales en países independientes establecido en 1989 representa "el marco legal más avanzado en lo que concierne a las aspiraciones y derechos indígenas sobre la tierra, al respeto socio-cultural en general y a cierto grado de autonomía” (Gasché, 2008, p. 2).

${ }^{7}$ López (2001, p. 3) menciona que aun cuando no es reconocido ampliamente en leyes o constituciones, el término "interculturalidad" es planteado y discutido por primera vez en la década de 1980, en una reunión continental convocada conjuntamente por la Unesco y el Instituto Interamericano, ante las inquietudes levantadas por un proyecto educativo realizado en Venezuela con población indígena por Monsonyi y González en la década de 1970 (Ferrão, 2010, p. 345).
}

82 - Breve panorama histórico de la educación intercultural en América Latina Jocelin Venegas Martínez, Luz Marías Stella Moreno Medrano. DIDAC 76 (2020): 77-85 
ción de sociedades más equitativas e igualitarias, pues no cuestiona las "reglas del juego" (Tubino, 2005 , p. 3). Este tipo de interculturalidad también ha sido denominada interculturalidad armónica, pues no considera las relaciones asimétricas de poder y se limita a ver la educación para la interculturalidad como "punto de llegada y no de partida" (Bertely, 2013, p. 46). Como consecuencia, la educación intercultural es una estrategia que reproduce las desigualdades sociales bajo un discurso romántico de valoración de las diferencias, pero sin ningún impacto real en el reconocimiento de los pueblos indígenas como sujetos de derechos.

Por otro lado, la interculturalidad crítica es una propuesta ético-política, cuyo objetivo es la construcción de una sociedad distinta a la que habitamos, en la que todas las voces deben ser escuchadas y valoradas en "condiciones de respeto, legitimidad, simetría, equidad e igualdad" (Walsh, 2010, p. 4). Para que esto se logre es necesario "no sólo cambiar las relaciones, sino también las estructuras, condiciones y dispositivos de poder que mantienen la desigualdad, inferiorización, racialización y discriminación" (Walsh, 2010, p. 4) ${ }^{8}$ De esta forma, la educación interculturalidad crítica es explícita y profundamente política.

En sintonía con estas ideas, Tubino señala que la interculturalidad crítica va encaminada hacia una "democracia radical pues es una democracia inclusiva de la diversidad alternativa al carácter occidental" (2005, p. 2). Además, sostiene que para que una sociedad de este tipo pueda empezar a construirse, habría que poner sobre la mesa las condiciones de asimetría presentes en el mundo, es decir, saber nombrarlas, reconocerlas y denunciarlas para lograr traer la historia al presente y comenzar a analizar las deudas históricas con los pueblos que han sido marginados. Es por esto que se considera que la interculturalidad crítica es aún un proceso en cons- trucción, no existe de facto; requiere la participación de toda la sociedad, no sólo de las poblaciones indígenas y afrodescendientes, a quienes históricamente ha estado dirigida en América Latina. Así pues, se trata de una tarea ciudadana que implica también el reconocimiento de los privilegios de los grupos mayoritarios (Ferrão, 2010, p. 349).

En este sentido, el gran reto de la educación intercultural en el siglo XXI es traer a la luz los procesos de colonización por los que ha pasado Abya Yala, para lograr deconstruirlos y conocer los mecanismos que usa el sistema para seguir oprimiendo a los pueblos indígenas. En el ámbito educativo en específico, Catherine Walsh (2013) considera la interculturalidad crítica como una herramienta pedagógica para cuestionar el sistema, las relaciones de poder y las formas en que nos vinculamos con los otros.

Así, se abre la posibilidad de construir nuevas realidades sociales en donde otros conocimientos, prácticas, saberes y epistemologías sean reconocidas y tengan un espacio que aportar de la misma forma en que otras culturas lo han hecho durante siglos. La educación intercultural crítica sigue siendo un tema pendiente en América Latina y debe ir más allá de las aulas para conquistar los espacios públicos y privados, así como a sectores de la sociedad que no han logrado cuestionar el racismo y el clasismo que se ha formado en nuestras mentes como consecuencia de la historia de conquista y colonización.

La educación intercultural crítica es, pues, una posibilidad de construirnos de manera horizontal entre las distintas formas de ver y entender el mundo (indígenas, no indígenas, afrodescendientes, homosexuales o heterosexuales, de clase trabajadora o clase adinerada) y, al mismo tiempo, de posicionarnos en la búsqueda por la igualdad de derechos de los más desprotegidos, como los pueblos indígenas $\mathrm{y}$ afrodescendientes. I

\footnotetext{
${ }^{8}$ Bolivia y Ecuador son países pioneros en introducir esta perspectiva en sus sistemas políticos, pues han emprendido acciones encaminadas a la creación de condiciones más justas y equitativas para los pueblos originarios, apostando a la construcción de Estados plurinacionales (Ferrão, 2010 , p. 350). Esto implica, además de reconocer las lenguas propias y las tradiciones de cada etnia, reconocerlos como actores y sujetos de derechos, darles autonomía y hacerlos partícipes de las decisiones del Estado.
} 
Abram, M. (2004). Estado del arte de la educación bilingüe intercultural en América Latina. Washington. Recuperado de http://www.humanas.unal.edu.co/colantropos/files/27 14/7941/3665/Estado_del_arte_de_la_educacion_bilingue_intercultural_en_America_Latina_.pdf

Ajagán, L. (2017). Interculturalidad: aproximaciones y perspectivas del concepto. Seminario Derechos de la Infancia e Interculturalidad. Desafíos para la Educación. Perspectivas, Tensiones, Juicios y Prejuicios Respecto de sus Interpretaciones. Universidad de Concepción, Chile.

Apodaca, E. (2009). Acerca del multiculturalismo, la educación intercultural y los derechos indígenas en las Américas. Revista de Investigación Educativa, 9, 1-11. Recuperado de https://www.redalyc.org/pdf/2831/283121714003.pdf

Bertely, M. (2013). Debates conceptuales sobre educación multicultural e intercultural. En M. Bertely, G. Dietz \& M. G. Díaz Tepepa (Coords.), Multiculturalismo y educación: 2002-2011 (pp. 41-79). México: ANUIEs, COMIE.

Briceño, G. (2019). De las políticas educativas de interculturalidad al aula: modelos pedagógicos de aplicación. En M. A. Paz \& I. Flores (Coords.), Experiencias en educación e interculturalidad. Reflexiones metodológicas y nuevos desafios teóri$\cos$ (pp. 113-137). Guadalajara, México: UdeG.

Chirinos, A. \& Zegarra, M. (2004). Educación indígena en Perú. Estudios sobre la Educación Universitaria Peruana, 4(92).

Díaz-Couder, E. (1998). Diversidad cultural y educación en Iberoamérica. Revista Iberoamericana de Educación, 17, 11-30.

Dietz, G. (2012). Multiculturalismo, interculturalidad y diversidad en educación. Una aproximación antropológica. México: FCE.

Fajardo, D. (2011). Educación intercultural bilingüe en Latinoamérica: un breve estado de la cuestión. LiminaR. Estudios Sociales y Humanísticos, 9(2), 15-29.

Ferrão, V. (2010). Educación intercultural en América Latina: distintas concepciones y tensiones actuales. Estudios Pedagógicos, $\mathrm{xxxvI}(2), 333-342$.

Fondo de las Naciones Unidas para la Infancia (Unicef). (2009). Atlas sociolingüistico de lenguas indigenas en América Latina. Cochabamba: funproeib Andes. Recuperado de https://www.unicef.org/honduras/tomo_1_atlas.pdf

Gasché, J. (2008). La motivación política de la educación intercultural indígena y sus exigencias pedagógicas. ¿Hasta dónde abarca la interculturalidad? En M. Bertely, J. Gasché \& R. Podestá (Coords.), Educando en la diversidad. Investigaciones y experiencias educativas interculturales y bilingües (pp. 367-397). Quito, Ecuador: Abya Yala.
López, L. (1998). La Eficacia y validez de lo obvio: Lecciones aprendidas desde la evaluación de procesos educativos bilingües. Revista Iberoamericana de Educación, (17), 51-89.

López, L. (2001). La cuestión de la interculturalidad y la educación latinoamericana. Séptima Reunión del Comité Regional Intergubernamental del Proyecto Principal de Educación en América Latina y el Caribe. Unesco. Documento de Apoyo. Recuperado de http://red.pucp.edu.pe/ ridei/files/2011/08/731.pdf

Loyo, E. (1996). La Empresa Redentora. La Casa del Estudiante Indígena. HMex, XLVI(1), 99-131.

Moya, R. (1998). Reformas educativas e interculturalidad en América Latina. Revista Iberoamericana de Educación, 17, 105-187.

Santoyo, M. y Arellano, J. (1997). El Instituto Lingüístico de Verano y el Protestantismo en México. Convergencia Revista de Ciencias Sociales, (14), 231-262.

Sartorello, S. (2009). Una perspectiva crítica sobre interculturalidad y educación intercultural bilingüe: el caso de la Unión de Maestros de la Nueva Educación para México (UNEM) y educadores independientes en Chiapas. Revista Latinoamericana de Educación Inclusiva, 3(2), 77-90.

Schmelkes, S. (2003). Educación intercultural. Reflexiones a la luz de experiencias recientes. Sinéctica, Revista Electrónica de Educación, 23, 26-34.

Schmelkes, S. (2004). La educación intercultural: un campo en proceso de consolidación. Revista Mexicana de Investigación Educativa, 9(20), 9-13.

Tubino, F. (2005). La interculturalidad critica como proyecto ético-politico. Trabajo presentado en el Encuentro Continental de Educadores Agustinos, Lima. Recuperado de https://oala. villanova.edu/congresos/educacion/lima-ponen-02.html

Walsh, C. (1998). La interculturalidad en la educación. Lima, Perú: Unicef.

Walsh, C. (2005). Interculturalidad, conocimientos y decolonialidad. Signo y Pensamiento, XIV (46), 39-50.

Walsh, C. (2010). Interculturalidad crítica y educación intercultural. En Construyendo interculturalidad critica (pp. 7596). La Paz, Bolivia: Instituto Internacional de Integración del Convenio Andrés Bello.

Walsh, C. (2013). Pedagogías decoloniales, prácticas insurgentes de resistir, (re) existir y (re)vivir. Tomo I. Quito: Abya Yala.

Zolla, C. \& Zolla Márquez, E. (2010). Los pueblos indígenas de México. 100 preguntas. México: Unam. Recuperado de http:// www.nacionmulticultural.unam.mx/100preguntas/index.html

BibliografíA RECOMENDADA

Banco Mundial. (2015). Latinoamérica indígena en el siglo xxi. Primera década. Washington, D. C.: Banco Mundial. Recuperado de http://documents.worldbank.org/curated/ en/541651467999959129/pdf/98544-WP-P 148348 -Box394854B-PUBLIC-Latinoamerica-indigena-SPANISH.pdf
Dietz, G. (2016). Presentación. En G. Briceño \& T. Salzman (Coords.), Multiculturalismo y educación intercultural en México: realidades y desafios. Guadalajara, México: DAAD, CIPS/SEJ.

Dietz, G. (2017). Interculturalidad: una aproximación antropológica. Perfiles Educativos, 39(156), 192-207.

84 - Breve panorama histórico de la educación intercultural en América Latina

Jocelin Venegas Martínez, Luz Marías Stella Moreno Medrano. DIDAC 76 (2020): 77-85 
Durin, S. (Coord.). (2010). Etnicidades urbanas en las Américas. Procesos de inserción, discriminación y politicas multiculturalistas. México: CIEsas, Escuela de Graduados en Administración Pública y Política Pública del Tecnológico de Monterrey. Recuperado de https://www.academia. edu/37459365/_Etnicidades_urbanas_en_las_Am\%C3\%A9ricas._Procesos_de_inserci\%C3\%B3n_discriminaci\%C3\%B3n_y_pol\%C3\%ADticas_multiculturalistas_S\%C3\%A9verine_Durin_Coord._CIESAS_EGAP_ ITESM_M\%C3\%A9xico_ISBN_978-607-486-098-6

Ejército Zapatista de Liberación Nacional (EZLN). 2015. El pensamiento crítico frente a la hidra capitalista I. Participación de la Comisión Sexta del ezLN. México: EzLN.

Fanon, F. (1963). Los condenados de la Tierra. México: FCE.

Freire, P. (28 de mayo de 1978). Entrevista: Paulo Freire: La educación es siempre un quehacer político. El País. Recuperado de https://elpais.com/diario/1978/05/20/sociedad/264463223_850215.html

Gasché, J. (2010). De hablar de la educación intercultural a hacerla. Mundo amazónico, 1, 111-134.

Gónzalez, J. (Ed.). (2019). Multiculturalismo e interculturalidad en las Américas. Canadá, México, Guatemala, Colombia, Bolivia, Brasil, Uruguay. Bogotá: Cátedra Unesco Diálogo Intercultural, Universidad Nacional de Colombia. Recuperado de https:/www.academia.edu/40115208/MULTI-
CULTURALISMO_E_INTERCULTURALIDAD_EN_ LAS_AM\%C3\%89RICAS

Instituto Nacional de los Pueblos Indígenas (INPI). (5 de diciembre de 2018). Instituto Nacional de los Pueblos indígenas (INPI). Gobierno de México. Recuperado de https:// www.gob.mx/inpi/articulos/instituto-nacional-de-los-pueblos-indigenas

Leyva, X., et al. (2015). Prácticas otras de conocimiento(s). Entre crisis, entre guerras. Tomo I. México: Cooperativa Editorial Retos.

Leyva, X., et al. (2015). Prácticas otras de conocimiento(s). Entre crisis, entre guerras. Tomo II. México: Cooperativa Editorial Retos.

Leyva, X., et al. (2015). Prácticas otras de conocimiento(s). Entre crisis, entre guerras. Tomo III. México: Cooperativa Editorial Retos

Pacari, N. (2007). Todo puede ocurrir. México: unam.

Robles, S. \& Cardoso R. (Coords.). (2007). Floriberto Díaz. Escrito. Comunalidad, energía viva del pensamiento mixe. México: UnAM.

Walsh, C. (2012). Interculturalidad y (de)colonialidad: perspectivas críticas y políticas. Visão Global, Joaçaba, 15(1-2), 61-74.

Walsh, C. (2017). Pedagogias decoloniales, prácticas insurgentes de resistir, (re) existir y (re)vivir. Tomo II. Quito: Abya Yala.

\section{SEMBLANZAS}

Jocelin Venegas Martínez. Estudió la licenciatura en psicología en la Universidad Nacional Autónoma de México (UNAM) y es maestrante por el Departamento de Investigaciones Educativas del Centro de Investigación y de Estudios Avanzados (Cinvestav). Tiene amplia experiencia en la elaboración de objetos de aprendizaje para bachillerato y ha sido docente de ese mismo nivel educativo. Actualmente colabora como asistente para el fortalecimiento de la calidad académica en la licenciatura de Pedagogía de la Universidad Iberoamericana. En el campo educativo le interesan temas vinculados con la interculturalidad, la equidad y el desarrollo comunitario.

Luz María Stella Moreno Medrano. Profesora investigadora en el Departamento de Educación de la Universidad Iberoamericana y coordinadora de la licenciatura en Pedagogía. Después de haber sido docente en una primaria indígena en la sierra Tarahumara durante dos años (1994-1996), estudió la licenciatura en Educación en la Universidad de Monterrey (UDEM) con una especialización en Desarrollo Comunitario. Posteriormente, realizó sus estudios de maestría en la Escuela de Educación de la Universidad de Harvard. Sus líneas de investigación están dirigidas a la interculturalidad, la educación en contextos vulnerados y más recientemente en acompañar los procesos de formación de docentes y directivos. Concluyó su doctorado en la Universidad de Cambridge con la investigación etnográfica: Experiencias escolares de niños indígenas en escuelas urbanas en Jalisco. Entre sus publicaciones más recientes destacan: Historias de aqui y de allá. Relatos desde la perspectiva bicultural de niños migrantes y The Construction of Resilience: Voices of Poor Children in Mexico. 